\title{
EFECTO DEL FEEDBACK CORRECTIVO DIRECTO Y METALINGÜISTICO EN EL PROCESO DE ESCRITURA DE UNA L2 EN EL CONTEXTO ESCOLAR: ESTUDIO PILOTO
}

\section{THE EFFECT OF DIRECT AND METALINGUISTIC WRITTEN CORRECTIVE FEEDBACK IN THE SCHOOL CONTEXT: A PILOT STUDY}

Jorge Lillo Durán*

\section{RESUMEN}

Desde los inicios de la investigación en Adquisición de Segundas Lenguas (ASL), los estudios de Feedback Correctivo Escrito (FCE) buscaron respuesta a diversas preguntas en torno al FC y en especial al escrito: las razones para corregir los errores, cuáles errores corregir, cuándo corregirlos, cómo corregirlos y por quién. Sin embargo, los estudios iniciales no se concentraron en la pregunta que es de vital importancia en esta temática el día de hoy, es decir, si el FC tiene el potencial de contribuir al desarrollo de la lengua meta (BITCHENER \& FERRIS, 2012). En este artículo se informa de los resultados de un estudio piloto que aborda la efectividad del FC escrito. Los hallazgos revelan que los grupos experimentales obtuvieron mejores resultados que el grupo control en el postest escrito; sin embargo debido a ciertas limitaciones del estudio piloto, los resultados hay que visualizarlos con cautela. Palabras-claves: adquisición de segundas lenguas; errores gramaticales; feedback correctivo escrito.

\section{ABSTRACT}

Since the early research in Second Language Acquisition (SLA), studies of Written Corrective Feedback (WCF) sought to answer several questions about CF and especially WCF: reasons to correct errors, which errors to correct, when to correct, how to correct and by whom. However, initial studies did not focus on the question that is vital in this area today, that is, if CF has the potential to contribute to the development of the target language (BITCHENER \& FERRIS, 2012). This article reports the results of a pilot study that addresses the effectiveness of WCF. The findings reveal that the experimental groups outperformed the control group in the written posttest; however, due to certain limitations of the pilot study, the results need to be revised with caution.

Keywords: accuracy; second language acquisition; written corrective feedback.

\footnotetext{
*UCSC, Universidad Católica de la Santísima Concepción, Chile. jlillo@ucsc.cl
} 


\section{INTRODUCCION}

El feedback acerca de la escritura en una segunda lengua (L2) ha sido considerado uno de los temas relevantes en la investigación asociada al aprendizaje y adquisición de una L2 en las últimas décadas. Este está dirigido a retroalimentar tres ámbitos bien definidos: el contenido, la organización y la lengua. El feedback que se preocupa de corregir los errores de lengua, el denominado feedback correctivo, es el que ha concitado el interés de los académicos y que además ha generado una gran controversia, principalmente en torno a su efectividad (TRUSCOTT, 1996; FERRIS, 1999). Mucho se ha investigado en las últimas décadas para examinar esta temática de interés académico y aunque en los últimos años una serie de estudios con una metodología robusta ha entregado resultados auspiciosos, la investigación primaria dominante se ha realizado en contextos de English as a Second Language (ESL) más que de English as a Foreign Language (EFL). Los estudios de este segundo enfoque son virtualmente limitados y prácticamente inexistentes en nuestro contexto de aprendizaje; por consiguiente, los profesores de lenguas todavía no tienen lineamientos claros o información relevante a su entorno pedagógico para encontrar en el FC una solución a la corrección de los errores de escritura en una segunda lengua. En el siguiente artículo se informa de aspectos teóricos relevantes para la corrección de los errores escritos en una segunda lengua que pueden entregar mayor claridad a profesores e investigadores de qué elementos considerar a la hora de tomar ciertas decisiones en torno al FC. Se presenta el diseño y los hallazgos de un estudio piloto realizado en un colegio bilingüe chileno en relación con el FC correctivo escrito y sus repercusiones para el aprendizaje de una segunda lengua.

\section{FUNDAMENTACION TEORICA}

\subsection{La efectividad que tiene el Feedback Correctivo Escrito}

El feedback correctivo es una estrategia asociada regularmente a la adquisición de la habilidad de producción oral (SHEEN, 2011); sin embargo, es también una herramienta valiosa que utilizan los profesores como estrategia de aprendizaje y actividad de aula permanente en el desarrollo de otras habilidades de la lengua. El impacto que el feedback correctivo escrito tiene en la ASL ha generado gran interés en la comunidad académica y demostrado ser un polo de investigación interesante por el potencial que tiene en el mejoramiento del proceso de adquisición de la len- 
gua, pero a la vez, ha generado confusión y discusión entre los especialistas tanto por las diferencias en los resultados obtenidos como por su efectividad.

Hay un hito en la literatura que sin duda ha sido el que ha impulsado la investigación de la última década, a saber, la discusión académica entre Truscott y Ferris desde fines de los años 90 (TRUSCOTT 1996; 1999; 2004; 2007; 2009; FERRIS, 1999; 2002; 2004; 2010). Truscott (1996) en su famoso artículo "The Case Against grammar correction in L2 Writing Classes" declara que la entrega de feedback correctivo escrito a los estudiantes de inglés como segunda lengua no es efectivo y que además puede ser dañino para el desarrollo de la escritura (MILTON, 2006; BITCHENER, 2008; BITCHENER \& KNOCH 2008; ELLIS et al., 2008; SHEEN, 2011), argumentando que, primero, no existe evidencia empírica que sustente el supuesto de que la corrección de errores escritos ayuda a los hablantes de una segunda lengua a mejorar su precisión lingüística escrita, sino que por el contrario, existe evidencia robusta para sugerir su inefectividad. Continúa Truscott (2004) en su argumentación en contra del FC señalando que, desde un punto de vista teórico, la corrección de los errores no puede contribuir al desarrollo de la competencia de una L2 o influir en el orden natural y la secuencia de adquisición, ya que esta es dependiente del syllabus interno del estudiante. Finalmente, discute que la provisión de FC por parte de los profesores crea muchos problemas prácticos, ya sea por la inconsistencia en la manera en que el feedback se entrega, en por la ansiedad que provoca (SHEEN, 2011) o por la toma de decisiones de cuáles errores corregir. En su respuesta, Ferris (1999) argumenta que la base de investigación a la que Truscott hace referencia es demasiado limitada y conflictiva en sus resultados, por lo tanto debe haber cierta moderación en los comentarios hasta que exista mayor consistencia en la investigación, agregando que si la corrección es clara y consistente debería funcionar (ELLIS et al., 2008). También agrega Ferris que Truscott ha subestimado el potencial de la evidencia arrojada por algunos estudios en el efecto positivo de la corrección de la gramática (FERRIS, 2004) y también puntualiza que si bien es cierto que el deseo de corrección de los estudiantes no puede estar sobre la toma de decisiones de los profesores, como señala Truscott, éste no se puede desechar u omitir tan fácilmente.

A pesar del debate entre Ferris y Truscott en relación a la corrección de los errores, que por cierto ha durado más de una década, existen dos puntos en los que estos autores muestran acuerdo: el primero en relación a que la investigación en la corrección de los errores en la escritura de una L2 es insuficiente y segundo, que faltan estudios con diseños metodológicos robustos. 


\subsection{Decisiones en torno al uso del Feedback correctivo escrito}

En el apartado anterior se dan argumentos tanto a favor como en contra del uso del FC en la enseñanza de la escritura en una L2. Sin embargo, incluso entre los defensores del FC escrito, algunas situaciones en relación con el valor de la corrección de los errores permanecen no resueltas. A continuación, se revisan dos de las temáticas fuertemente debatidas en ese aspecto, (1) la eficacia diferenciadora de diversas metodologías de FC, y (2) la significancia de diferentes tipos de errores para el FC.

\subsubsection{Métodos para la corrección de los errores escritos}

El FC en la escritura de los estudiantes de una segunda lengua puede adoptar muchas formas diferentes. Las Metodologías de la corrección de los errores escritos pueden variar, por ejemplo, con respecto a su enfoque o al medio de la entrega, entre otras. En este apartado se discutirá la pertinencia del FC escrito, la temporalidad de su aplicación, las estrategias comúnmente utilizadas y la responsabilidad de la entrega.

\subsubsection{La validez de la corrección de los errores de los estudiantes}

Existe hoy en día mayor consenso en torno a que los estudiantes avanzan en el desarrollo de su interlengua a través de la prueba constante de sus hipótesis y desde esa perspectiva el FC les brinda una oportunidad para tal proceso (SHEEN, 2011). Pero, en los años iniciales de investigación en FC, el foco estaba centrado en las razones para corregir los errores más que en si puede funcionar o tener algún papel importante en el proceso de adquisición de una segunda lengua (BITCHENER \& FERRIS 2012). Aun así, para investigadores como Corder (1973), la corrección era importante porque se esperaba ayudar a los estudiantes a identificar sus propios errores y descubrir las funciones y limitaciones de las estructuras sintácticas y léxicas de la lengua meta. Además, numerosos estudios han demostrado que los estudiantes desean ser corregidos (SHEEN, 2011), lo que entrega mayor respaldo para corregir los errores de parte de los propios estudiantes. Hendrickson (1978), Crookes y Chaudron (1991) argumentan acerca de la utilidad del FC, señalando estos últimos que los estudiantes necesitan feedback para diferenciar el uso aceptable e inaceptable de la lengua. 


\subsubsection{Cuándo se deben corregir los errores escritos}

Un tema crucial en la corrección de los errores se refiere a si deben ser corregidos inmediatamente después que ocurren o diferir el FC para más tarde (SHEEN, 2011). Hendrickson (1978) se dio cuenta que los profesores de lengua ya habían rechazado la tesis de evitar los errores a toda costa durante el enfoque audiolingual y aceptaban un amplio rango de ellos y solamente trataban aquellos que resultaban más problemáticos. Hoy en día existe mayor consenso en que el FC tiene un rol importante en la precisión gramatical, pero algunos metodólogos sienten que no tiene un lugar relevante en la fluidez, argumentando que los estudiantes no deben ser interrumpidos cuando hablan (HARMER, 2007). Estos investigadores recomiendan corregir los errores en forma diferida hasta que la actividad comunicativa haya sido completada. Este punto es particularmente importante en el FC escrito, ya que en prácticamente todas las ocasiones se realiza en forma diferida, lo que conlleva ciertos beneficios desde el punto de vista del procesamiento de la información, porque el estudiante tiene mucho más tiempo para reflexionar acerca de los errores cometidos y para analizar la distancia que existe con la lengua meta.

\subsubsection{Cómo se deben corregir los errores escritos}

Hendrickson (1978) informa acerca de la importancia de investigar el valor de la corrección indirecta y directa, los métodos de corrección sistemáticos y la entrega de feedback selectivo. Hoy en día, en relación al feedback selectivo, todavía existe diferencia entre la corrección focalizada (limitada a una o dos categorías de errores) y la no-focalizada (tratamiento de un amplio rango de errores). Aun cuando algunos metodólogos prefieren la corrección no-focalizada, estudios recientes han demostrado que la corrección focalizada es muy efectiva (ELLIS et al., 2008). En cuanto a las estrategias, las que mayoritariamente han sido objeto de discusión e investigación son el FC directo e indirecto (SHEEN, 2011). La corrección directa considera la entrega de la forma correcta, mientras que la indirecta es una indicación de que hubo algún error, pero sin entregar la forma correcta. Existe mayor preferencia entre los expertos en producción escrita por el feedback indirecto, argumentando que este tipo de corrección es más efectiva porque requiere que los estudiantes se involucren en actividades de resolución de problemas y de autocorrección porque además demanda mayor procesamiento de la lengua (FERRIS, 1999; LALANDE, 1982; BITCHENER \& KNOCH, 2008). Sin embargo, los defensores del feedback 
correctivo directo (CHANDLER, 2003) argumentan que el feedback indirecto entrega a los estudiantes información incompleta para resolver los errores complejos. Aun más, Chandler (2003) informa que mientras el FC directo capacita a los estudiantes a internalizar instantáneamente la forma correcta, los estudiantes a los que se les corrige los errores en forma indirecta no son capaces de saber si sus propias correcciones hipotéticas son realmente precisas. Por otra parte, Bitchener y Knoch (2010b) sugieren que solo la corrección directa es capaz de ofrecer a los estudiantes el tipo de información explícita necesaria para probar sus hipótesis acerca de la lengua meta. Estudios recientes (BAKER \& BRICKER, 2010) han encontrado evidencia empírica que sostiene que los estudiantes corrigieron sus textos en forma más precisa cuando la corrección fue directa; además, los estudiantes encontraron dificultades para identificar la corrección indirecta e identificaron en los casos de corrección indirecta más extrema los peores resultados en la precisión gramatical en la revisión de los textos escritos.

\subsubsection{Responsabilidad en la corrección de los errores}

La respuesta a esta interrogante ha sido considerada en forma intuitiva más que como resultado de la investigación. Hendrickson (1978) informa que los efectos relativos de la corrección de los profesores, la corrección de pares y la autocorrección podrían ser argumentados o refutados mediante una serie de experimentos controlados. Hendrickson (1980) sugiere que, aunque los profesores son los que tienen la responsabilidad de corregir los errores de sus estudiantes, no debiera ser siempre el método dominante, permitiendo a los estudiantes corregir sus propios errores mediante la autocorrección y la corrección de pares. De estos dos últimos métodos, existe soporte teórico para sustentar que el primero de ellos, la autocorrección, estimula a los estudiantes a revisar su interlengua, a notar la brecha que existe con la lengua meta y a probar sus hipótesis (SHEEN, 2011). Sin embargo, este método no está exento de controversia debido a que resulta un tanto problemático en términos prácticos, porque los estudiantes prefieren que los profesores sean los que corrijan y además podrían no estar capacitados para autocorregirse si no tienen el suficiente conocimiento lingüístico o si los errores están fuera del estadio correspondiente, en términos de Pieneman (1998). En cuanto a la corrección de pares, muchos académicos consideran que este método es importante (FERRIS, $2003 ;$ 2004); sin embargo, aunque ha sido practicado en los últimos años, no ha sido sometido a suficiente comprobación empírica. Además, los profesores necesitan asegurarse de que los estudiantes tengan el conocimiento lingüístico para desar- 
rollar esta tarea, de lo contrario podría no conducir a la corrección de los errores (HYLAND \& HYLAND, 2006).

\subsubsection{Diferentes tipos de errores en ASL}

Los estudiantes de segundas lenguas cometen errores de comprensión y producción, los que son considerados como estructura no deseadas de la lengua (ELLIS, 1994). Corder (1967) define el error como una desviación sistemática y consistente que demuestra que el estudiante aprende y que caracteriza su interlengua. Corder introdujo la distinción entre "errors" (en competencia) y "mistakes" (en actuación); esta distinción dirigió la atención de los investigadores de ASL a los errores de competencia y estableció un marco más concentrado. Así, en la década de los 70, los investigadores comenzaron a examinar los errores de competencia de los alumnos y trataron de explicarlos. Corder mencionó para quiénes y para qué fines el estudio de los errores sería significativo y en consecuencia la investigación se dirigió no solo al entendimiento de los errores en sí mismo, sino también a mejorar la competencia lingüística. Burt (1975) define los errores como globales o locales. Los primeros son aquellos que conllevan problemas de comunicación al interferir con la comprensión de los enunciados (e.g. errores léxicos) mientras que las violaciones lingüísticas mínimas que no afectan la intención comunicativa del mensaje son categorizadas como errores locales (e.g. errores morfológicos). Para Sheen (2011) los errores globales interfieren con la comprensión y los errores locales no impiden la inteligibilidad de las oraciones. O en términos de Bitchener y Ferris (2012) errores que afectan o no la comunicación significativamente. Hendrickson (1978) recomienda a los profesores solamente corregir los globales porque son estos los que impiden la comunicación. Krashen $(1981 ; 1982 ; 1985)$ no reconoce que el FC tenga algún rol importante en el proceso de adquisición de una L2, pero que podría tener algún valor en la medida que los estudiantes puedan monitorear la producción del conocimiento adquirido. En ese contexto, Krashen menciona que el efecto facilitador del FC se limita solamente a reparar estructuras simples (e.g. la tercera persona singular - s del inglés) y que por consiguiente el FC solamente debería focalizarse en este tipo de errores.

Sheen (2011) y Bitchener \& Ferris (2012) distinguen también entre aquellos errores que tienen efectos estigmatizantes en los lectores o auditores (errores que ofenden a los lectores o auditores de la lengua meta) y los errores más o menos frecuentes que ocurren en la escritura de los estudiantes (la frecuencia de un tipo de error en particular en relación con otro tipo). Finalmente, Ferris (1999; 2002) 
distingue entre errores tratables versus no tratables. Para Ferris, los primeros son aquellos que ocurren en un patrón de acuerdo a alguna regla y los segundos son aquellos para los que no hay reglas que los estudiantes puedan consultar para evitarlos o repararlos. Precisa además que los errores idiosincráticos (no-idiomáticos) son intratables (e.g. errores léxicos), y aquellos que corresponden a estructuras determinadas por patrones y reglas son tratables (e.g. errores de artículos). Ferris sugiere que el FC debiera ser más exitoso cuando se dirige a reparar los tratables.

A pesar de los consensos, no siempre es tan fácil ver qué criterios utilizar, no siempre es tan simple determinar si un error es tratable o no (SHEEN, 2011). De hecho existen problemas con todas estas propuestas y no existe una clara base teórica para ninguna de ellas. Ellis (2009) argumenta, por ejemplo, que la dicotomía entre "mistakes" y "errors" no es tan estricta como Corder (1967) la presenta y afirma que la gravedad de un error es una cuestión de opinión personal (ELLIS, 2009). Aún más, él señala que no hay fundamentos teóricos sobre el que profesores e investigadores puedan decidir si un error es simple; o si es tratable o no. Para Van Beuningen (2010) solamente existe una distinción operacional, que es el contraste entre los errores gramaticales y los errores que existen fuera del dominio de la gramática.

A partir de los argumentos dados en los apartados anteriores y contrariamente a lo expuesto por Truscott $(1996 ;$ 2007), este artículo postula que el FC escrito es un elemento importante capaz de afectar las reglas que subyacen a los errores gramaticales (VAN BEUNINGEN, 2010) en forma positiva. En el estudio, se ha tratado de evitar las falencias de diseño a las que ha aludido Truscott (2007) y se ha incorporado e integrado lo aportado por los estudios de FC escrito en los últimos años (BITCHENER et al., 2005; BITCHENER, 2008; SHEEN, 2007, ELLIS et al. 2008).

\section{METODOLOGÍA DE TRABAJO}

Este estudio piloto es parte de un proyecto mayor que investiga el Feedback correctivo escrito y la adquisición de una L2. El objetivo principal fue el de comparar el efecto de dos modalidades de feedback correctivo escrito, a saber, la corrección directa y la corrección metalingüística en la adquisición del pasado simple en estudiantes de un nivel intermedio de English as a Foreign Language (EFL), al mismo tiempo que sirvió para probar instrumentos y métodos de análisis de resultados para el estudio principal. Las preguntas de investigación que se buscaron responder son las siguientes: 
1. ¿Cuál es el efecto que tiene el feedback correctivo escrito directo y metalingüístico en la adquisición del pasado simple en textos nuevos escritos en inglés?

2. ¿Cuál es el efecto diferenciador entre los dos tipos de FC escrito mencionados anteriormente en la adquisición del pasado simple en textos nuevos escritos en inglés?

\subsection{La estructura investigada}

Como se mencionara anteriormente, la estructura investigada en este estudio es el pasado simple. Se seleccionó utilizando dos criterios, el primero, basado en lo informado por los profesores que enseñan inglés en el colegio donde se realizó la investigación, quienes reconocen que esta estructura ha sido adquirida por algunos estudiantes pero no por otros; y el segundo, por lo encontrado en un análisis de errores sobre la escritura espontánea de los sujetos de la muestra, que arrojó como resultado que el pasado simple es una de las estructura con mayor porcentaje de errores en los textos escritos de los estudiantes.

El pasado simple tiene varias formas, por lo tanto, lo que realmente constituye el pasado simple necesita ser discutido. La forma del tiempo pasado, en comparación por ejemplo con el presente progresivo -ing (adquirida tempranamente por hablantes de de L1 y L2), requiere de un concepto desarrollado del pasado. En otras palabras, un morfema que tiene un significado en una sola categoría se puede considerar semánticamente más complejo que otro solo si tiene un significado que corresponda a un nivel mayor de desarrollo conceptual. Generalmente, su función representa una acción o estado finalizado en el pasado. Esta función, puede ser expresada a través de la cópula verbal (was-were), verbos regulares (aquellos que siguen una regla, eg. worked, asked) y los verbos irregulares (aquellos que no necesariamente siguen una regla eg. spoke, went). Puede ser expresada en forma activa o pasiva. Para este estudio, las formas del pasado simple consistieron en los verbos copulares, los verbos irregulares y los regulares en su forma activa.

\subsection{Método}

El método utilizado es similar al informado en el estudio de Sheen (2007) que considera dos grupos experimentales (FC directo y FC metalingüístico) y un grupo control. 


\subsubsection{Diseño de investigación}

El estudio piloto usó un diseño experimental. Se utilizaron dos condiciones experimentales y un grupo control, lo que se puede representar de la siguiente forma: FC directo $(\mathrm{N}=4)$, FC metalingüístico $(\mathrm{N}=5)$ y un grupo control $(\mathrm{N}=4)$. Los tres grupos completaron un pretest (T1), una tarea de edición del mismo texto (T2) y un postest (T3) inmediato. Los dos grupos experimentales recibieron corrección sobre sus errores escritos de acuerdo a la siguiente distribución:

\subsubsection{Feedback correctivo directo}

Esta corrección consistió en proveer al estudiante de la forma correcta, tachando el error y escribiendo la forma correcta, una palabra o morfema según sea el caso sobre la forma incorrecta.

feel

- How did you felt when you open the magic book?

\subsubsection{Feedback correctivo metalingüístico}

Esta corrección consistió en proveer la forma correcta en conjunto con una explicación metalingüística en la segunda lengua al pie de página.

- Your brothers where a little bit bad with you.

(1) In affirmative sentences use were as an auxiliary verb for the past tense.

\subsubsection{Grupo control}

El grupo control no recibió feedback correctivo, solamente un comentario general del contenido del texto como se indica a continuación.

- Ideas explained clearly but could bave been more developed.

La calendarización del estudio piloto se encuentra en la tabla 1. El estudio completo se realizó en el curso de tres semanas, durante el cual los estudiantes realizaron $\mathrm{T} 1, \mathrm{~T} 2$ y $\mathrm{T} 3$. 
Tabla 1. Calendario del Estudio

\begin{tabular}{|l|l|l|}
\hline 1 & $\bullet$ Pretest escrito (T1) & Semana 1 \\
\hline 2 & - Sesión de feedback escrito & Semana 2 \\
\hline 3 & - Revisión y edición del mismo texto (T2) & Semana 3 \\
& - Sesión de feedback escrito & \\
\hline
\end{tabular}

\subsubsection{La muestra}

La muestra del estudio incluyó a 13 estudiantes de $7^{\circ}$ básico de un colegio particular-bilingüe de la ciudad de Concepción, Chile, quienes han participado de un sistema de inmersión en inglés durante 5 años con un número aproximado de 3000 horas efectivas de exposición a la lengua inglesa y con 1,5 años de instrucción formal que incluye 256 horas de clases. Los sujetos fueron seleccionados al azar desde una población de 93 estudiantes y su edad fluctúa entre los 13 y 14 años. De acuerdo al número de horas de estudio de inglés se les puede considerar como estudiantes de un nivel intermedio de proficiencia.

\section{INSTRUMENTOS}

Para las tareas de composición escrita, los estudiantes escribieron una carta de 250 palabras. Se pensó en este tipo de texto porque crea las condiciones necesarias para elicitar la estructura objeto de estudio y está en concordancia con el tipo de textos y tareas que los estudiantes realizan habitualmente en sus actividades escolares como parte de su currículum. Para cada una de las instancias de evaluación los estudiantes escribieron un texto nuevo, cautelando que el grado de dificultad de la tarea fuera la misma. Para cada una de las tareas escritas se les pidió a los estudiantes información de identificación personal, así como también el nombre de su profesor de aula y su grupo curso, se les entregó un estímulo escrito, seguido de un recuadro para que pudieran organizar sus ideas y una hoja de papel con líneas para la composición. No se les exigió tiempo en la ejecución.

Para asegurar la autenticidad y validez de las tareas, un número de procedimientos fueron utilizados. Primero, en conjunto con el departamento de inglés del establecimiento, se realizó una revisión de los tipos de textos y tareas escritas a las que los estudiantes se ven enfrentados habitualmente. Posteriormente las tareas del estudio fueron sometidas a juicio de expertos por dos académicos, uno del Depar- 
tamento de Lenguas de la Universidad Católica de la Santísima Concepción y otro del Departamento de Idiomas Extranjeros de la Universidad de Concepción; sus comentarios fueron utilizados para mejorar la calidad de los instrumentos.

\section{PROCEDIMIENTOS}

\subsection{Grupos experimentales}

En la primera sesión, se les entregó a los estudiantes la primera tarea escrita (T1). Se les pidió que leyeran detalladamente las instrucciones y el estímulo para su realización. Posteriormente el investigador clarificó dudas en cuanto al propósito de la tarea y extrajo el vocabulario clave. Una vez que todos los sujetos tenían claro lo que se les pedía escribir, se les solicitó que organizaran sus ideas en el espacio correspondiente destinado para ello antes de comenzar con la escritura de la carta propiamente tal. Una vez finalizada la tarea, el texto escrito fue corregido por el investigador focalizándose principalmente en los errores de la estructura objeto de estudio. El tratamiento de feedback fue entregado a la sesión siguiente, de acuerdo al grupo de investigación (FD o FM). Una vez que los estudiantes recibieron el feedback correctivo en alguna de sus modalidades se les pidió que pusieran atención a las correcciones y que reescribieran el texto (T2). El texto escrito fue corregido por el investigador nuevamente focalizándose principalmente en los errores de la estructura objeto de estudio. El tratamiento de feedback fue entregado a la siguiente sesión y una vez revisada la corrección, se les pidió a los estudiantes que realizaran una nueva tarea escrita (T3).

\subsection{Grupo Control}

El grupo control realizó exactamente las mismas actividades en la misma secuencia que los grupos experimentales, sin embargo no recibió tratamiento de feedback correctivo alguno y solamente se les entregó un comentario general acerca del contenido del texto una vez finalizada la ejecución de las tareas escritas (T1, T2).

\section{RECOLECCIÓN Y ANALISIS DE DATOS}

Para analizar los efectos de los dos tipos de tratamiento (FC directo y FC metalingüístico) acerca del uso del pasado simple, se obtuvieron puntajes para cada 
una de las instancias de evaluación; pretest y postest escrito por parte del investigador.

Los puntajes de las tareas de escritura fueron calculados mediante el análisis de ocasiones obligatorias (ELLIS \& BARKHUIZEN, 2005). Todas las ocasiones obligatorias de uso del pasado simple fueron identificadas. Cada ocasión fue examinada para ver la correcta aplicación del pasado simple. La omisión de la aplicación de la estructura también fue considerada como error. El puntaje de precisión fue calculado para cada texto dividiendo el número de aciertos por el número total de ocasiones obligatorias multiplicado por el número de aplicación de la estructura en contextos no obligatorios.

n aciertos

$\mathrm{n}$ ocasiones obligatorias $+\mathrm{n}$ aplicaciones en contextos no obligatorios

Los puntajes de las tareas de escritura fueron analizados con ANOVA de medidas repetidas (3 grupos X 2 ocasiones) con pruebas de comparación múltiples Post Hoc de Tukey.

\section{RESULTADOS}

Una ANOVA de un factor revela que no existe una diferencia significativa en el pretest para las tres condiciones del estudio $(F(2,10)=0,128, p=0,881$.

La Figura 1 muestra una representación visual de la media de los puntajes de ambos tests (pretest-postest) para los dos grupos experimentales y para el grupo control. Los puntajes totales para los dos grupos experimentales aumentaron en el tiempo, mientras que para el grupo control disminuyó. La figura también muestra que el grupo que recibió feedback correctivo metalingüístico obtuvo mejores resultados que el grupo que recibió solo feedback correctivo directo. 


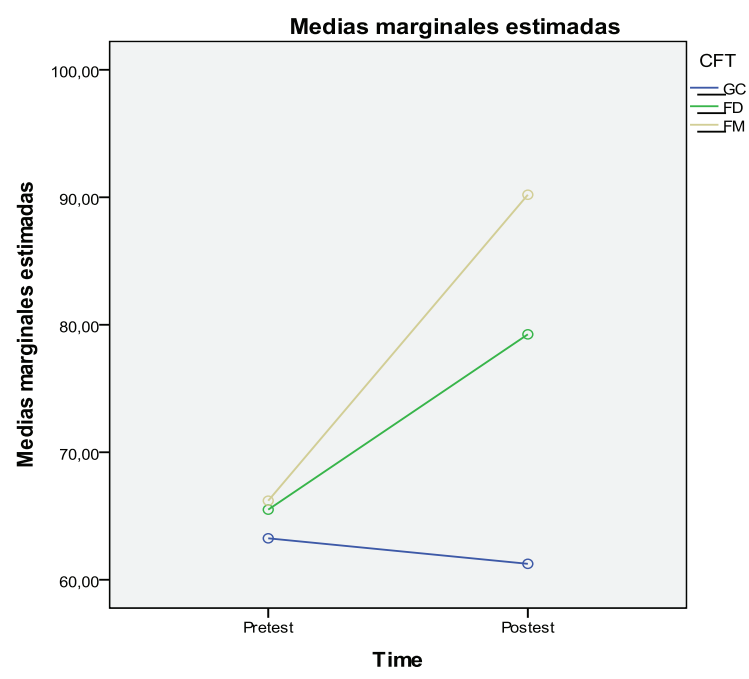

Figura 1. Medias grupales de los resultados de los Tests.

Para ver si las diferencias de los resultados de los grupos observados en la Figura 1 eran estadísticamente significativas, un análisis de varianza de medidas repetidas (ANOVA) fue realizado, con los resultados de los tests como variable dependiente y con el tiempo (pretest, postest) y el tratamiento de feedback correctivo como variables independientes.

Tabla 2. Pruebas de los efectos inter-sujetos

\begin{tabular}{|c|c|c|c|}
\hline Origen & g & F & Sig. \\
\hline Tratamiento de feedback correctivo & 2 & 4,806 &, 034 \\
\hline Error & 10 & & \\
\hline Tiempo & 1 & 22,191 &, 001 \\
\hline Tiempo*Tratamiento de feedback correctivo & 2 & 9,158 &, 005 \\
\hline Error(Tests) & 10 & & \\
\hline
\end{tabular}

Como se puede observar en la tabla 2, existe un efecto significativo para el tratamiento de $\mathrm{FC}$, esto demuestra que los grupos experimentales se desempeñaron mejor que el grupo control en los resultados totales de los tests cuando el tiempo fue controlado $(\mathrm{F}(2,10)=4,806 \mathrm{p}=, 034$. La tabla muestra que además hubo un efecto significativo de Tiempo $(\mathrm{F}(1,10)=22,191 \mathrm{p}=, 01)$ y una interacción 
también significativa entre Tiempo $*$ Tratamiento $(F(2,10)=9,158 \mathrm{p}=, 005$, indicando que los grupos actuaron en forma diferente entre ellos en el tiempo. Para detectar exactamente dónde se producen las diferencias entre los grupos, se realizó una comparación múltiple post hoc, utilizando el ajuste de comparaciones de Tukey. El análisis reveló que solo los estudiantes del grupo de FC metalingüístico tuvieron resultados estadísticamente significativos sobre el grupo control $(\mathrm{p}=, 028)$; no así los estudiantes del grupo de FC directo, quienes tuvieron mejores resultados, pero que no logran ser estadísticamente significativos $(\mathrm{p}=, 200)$. Además, los estudiantes del grupo metalingüístico obtuvieron mejores resultados que los del grupo de FC directo, pero la diferencia no es estadísticamente significativa $(p=, 520)$.

Para ver si el tratamiento de FC escrito tuvo un efecto en las diferencias de los resultados de los grupos experimentales y control en el postest, un análisis de varianza univariado (ANOVA) con pruebas post hoc de comparaciones múltiples fue realizado. El tratamiento de FC escrito es estadísticamente significativo para los resultados de los grupos experimentales en relación con el grupo control, $(F(2,10)$ $=11,670 ; \mathrm{p}=0,002$. El eta al cuadrado parcial es, 700 lo que sustenta el efecto del FC, es decir, el tratamiento de FC escrito es responsable en un $70 \%$ por la significancia estadística de los resultados del postest. Para detectar exactamente dónde se producen las diferencias entre los grupos, se realizó una comparación múltiple post hoc, utilizando el ajuste de comparaciones de Tukey. El análisis reveló que ambos grupos experimentales superaron significativamente al grupo control $(\mathrm{FCM} p=$ ,002 y FCD p =,042), el grupo de FC metalingüístico obtuvo mejores resultados que el grupo de FC directo; sin embargo la diferencia entre los resultados no es estadísticamente significativa.

\section{DISCUSIÓN}

La primera pregunta de investigación examinó el efecto que tiene el feedback correctivo escrito directo y metalingüístico en la adquisición del pasado simple en textos nuevos escritos en inglés. Para las tareas uno y dos se aplicaron los dos tipos de feedback correctivo, mientras que al grupo control solo se le entregó un comentario de carácter general sobre el contenido del texto. Los resultados demuestran que la escritura desde el punto de vista de la precisión gramatical mejora significativamente en los grupos experimentales, en contraste con el grupo control que prácticamente se mantuvo igual en ambas tareas escritas. Es decir, hubo un efecto importante del tratamiento de FC escrito por sobre el efecto de la repetición de 
las tareas escritas del grupo control en el aprendizaje del pasado simple del inglés. En cuanto a la segunda pregunta de investigación que examinó los efectos diferenciadores de dos estrategias de feedback correctivo directo en el aprendizaje, con o sin información metalingüística, la evidencia indica que el FC metalingüístico fue superior al FC directo, ya que solo el primero fue capaz de obtener resultados estadísticamente significativos por sobre los del grupo control cuando se analizan los resultados generales. El grupo de FC directo fue mejor que el grupo control, pero no logró establecer diferencias estadísticamente significativas en el tiempo. Sin embargo, al analizar solamente el postest, ambos grupos experimentales tuvieron resultados estadísticamente significativos por sobre los resultados del GC. Al comparar la diferencia que existe entre los dos tratamientos, no se encontraron diferencias significativas en los resultados generales, ni tampoco en los resultados del postest escrito. La estadística descriptiva indica que ambos grupos mejoraron sus resultados entre el pretest y el postest; sin embargo, el grupo de FC metalingüístico tuvo una ganancia en la media de 24 puntos mientras que el grupo de FC directo solo de 13.75. Al igual que lo expresado por Sheen $(2007 ; 2011)$, la superioridad del FC metalingüístico puede ser explicada de acuerdo al rol que tiene la conciencia en el aprendizaje de una L2 (SCHMIDT, 1994). El distingue dos niveles de conciencia en el aprendizaje, el notar (noticing) y un nivel superior que denomina entendimiento (understanding). Es factible que el FC directo y el FC metalingüístico sean capaces de promover el noticing, pero que solamente el metalingüístico lleve al estudiante a un nivel superior de procesamiento de la lengua, conducente a generar entendimiento. Finalmente, es importante destacar que en cuanto al número de estructuras objeto de análisis; mientras estudios anteriores se enfocaron en un rango mayor de categorías gramaticales, los resultados de este experimento demuestran el valor que tiene la focalización del tratamiento en una sola categoría gramatical. En este aspecto se corrobora los hallazgos de la investigación en FC escrito en los estudios previos (SHEEN, 2007; ELLIS et al., 2008; BITCHENER \& KNOCH, 2008). El estudio también revela que la oportunidad de practicar la escritura en una segunda lengua no tuvo un efecto significativo en la precisión gramatical de los textos de los estudiantes, este hallazgo es contrario a lo argumentado por Truscott (2004) quien sugiere que el aumento de precisión gramatical encontrado en estudios previos (e.g CHANDLER, 2003) se debe a la práctica y no a la corrección de errores. Además, la evidencia empírica del presente estudio contradice la recomendación realizada por Truscott (2004) acerca de que el tiempo dedicado a la corrección debería ser utilizado para realizar mayor práctica a través de actividades alternativas. 


\section{CONCLUSIONES E IMPLICACIONES DEL ESTUDIO}

La evidencia empírica que arroja este estudio sugiere que la corrección de los errores puede ser un medio efectivo para mejorar la precisión gramatical de la escritura en una segunda lengua; sin embargo, hay que tener cautela en cuanto a los resultados debido a las limitaciones que el estudio piloto conlleva: la muestra es pequeña y por lo tanto es difícil obtener resultados estadísticamente concluyentes. El estudio completo se realizó en tres semanas, es probable que esto no sea el tiempo suficiente para que se haya producido aprendizaje de la estructura objeto de estudio. El modelo de investigación utilizado considera dos tareas escritas y pretest-postest, lo que no permite observar el desempeño de los sujetos en torno a la estructura objeto de estudio en el tiempo. Si bien es cierto que la focalización del tratamiento en una estructura es altamente exitosa, difícilmente va a ser atractiva para que los profesores consideren esta opción en la sala de clases. Además puede resultar interesante comparar el uptake de diferentes tipos de errores (VAN BEUNINGEN et al., 2008) ya que Truscott (2007) dice que el FC solo podría ser efectivo en spelling correction. Estas limitaciones, de todas formas, han sido importantes para mejorar el estudio principal e incorporar ajustes en su diseño: la muestra ha sido aumentada significativamente a 80 sujetos. El modelo de investigación ha sido mejorado incorporando un ciclo de tareas más extenso que les permita a los sujetos escribir más. Se incorpora al diseño la revisión de textos, la generación de textos nuevos y una medida de evaluación a largo plazo. En el estudio principal se mantiene la focalización de los errores, pero se aumenta de una a dos las estructuras objeto de estudio.

Para concluir y de acuerdo a los resultados de este estudio y de la dirección que hoy día tiene la investigación en FC escrito, es posible realizar algunas recomendaciones pedagógicas. El FC que se entrega de manera sistemática acerca de los "errores lingüísticos" de los estudiantes puede mejorar su desempeño académico en la escritura de textos escritos. El estudio demuestra además que existe evidencia clara para recomendar a los profesores la entrega de FC acerca de una o dos categorías de errores porque es más efectivo, el uso de un tipo de FC con información metalingüística porque mejora el desempeño lingüístico de los estudiantes y los induce a notar sus errores al realizar un procesamiento de la lengua más profundo (notar y entender) lo que refleja adquisición. La evidencia también indica, al igual que los estudios de Bitchener (2008) y Bitchener y Knoch (2008), que una sola sesión de FC puede ser efectiva para desarrollar la precisión gramatical en el uso de una estructura gramatical, pero si los profesores son capaces de entregar feedback adicional en un mayor número de oportunidades, es posible aumentar la tasa de pre- 
cisión gramatical y disminuir el tiempo individual necesario para lograr un nivel de dominio sobre los errores. Finalmente, para confirmar la efectividad del FC escrito, es necesario realizar mayor investigación en contextos de clases con grupos intactos y en condiciones de aprendizajes más apropiadas a los contextos educacionales reales, es decir un enfoque de FC que además incorpore elementos innovadores en la corrección de los errores escritos y el proceso de producción escrita en una segunda lengua inserto en un solo diseño de investigación.

\section{REFERENCIAS BIBLIOGRAFICAS}

BAKER, W.; HANSEN BRICKER, R. (2010). The effects of direct and indirect speech acts on native English and ESL speakers' perception of teacher written feedback. System. v. 38, pp. $75-84$.

BURT, M. (1975). Error analysis in the adult EFL classroom. TESOL Quarterly. v. 9, pp. $3-63$.

BITCHENER, J. (2008). Evidence in support of corrective feedback. Journal of Second Language Writing. v. 17, pp. 102-118.

BITCHENER, J. et al (2005). The effect of different types of corrective feedback on ESL student writing . Journal of Second Language Writing, v.9 , pp. 227 - 258.

BITCHENER, J.; FERRIS, D. (2012) Written Corrective Feedback in Second Language cquisition and Writing. London: Routledge.

BITCHENER, J.; KNOCH U. (2008). The value of written corrective feedback for migrant and international students. Language Teaching Research. v. 12, pp. 409-431.

BITCHENER, J.; KNOCH U. (2010b). Raising the linguistic accuracy level of advanced L2 writers with written corrective feedback. Journal of Second Language Writing. v. 19, pp. 207-217.

CHANDLER, J. (2003). The efficacy of various kinds of error feedback for improvement in the accuracy and fluency of L2 student writing. Journal of Second Language Writing. v. 12 , pp. 267-296.

CORDER, S. P. (1967). The Significance of Learners' Errors. International Review of Applied Linguistics. v. 5, pp. $161-170$.

CORDER, S. P. (1973). Introducing Applied Linguistics. London: Penguin Books.

CROOKES, G.; CHAUDRON, C. (1991). Guidelines for classroom language teaching. In: Celce Murcia, M. (ed.), Teaching English as a Second or Foreign Language. Boston, MA: Heinle and Heinle, 46-67.

ELLIS, R. (1994). A theory of instructed second language acquisition. In: Ellis, N. (ed.), Implicit and Explicit Learning of Languages. Academic Press, San Diego. Pp. 79-114. 
ELLIS, R. (2009). A Typology of written corrective feedback types. ELT Journal. v. $63 \mathrm{n}^{\circ} 2$, pp. 97-107.

ELLIS, R.; BARKHUIZEN, G. (2005). Analyzing Learner Language. Oxford University Press, Oxford.

ELLIS, R. et al (2008). The Effects of Focused and Unfocused Written Corrective Feedback in English as a Foreign Language Context. System v. 36, pp. 353-371.

FERRIS, D. (1999). The case for grammar correction in L2 writing classes, a response to Truscott. Journal of Second Language Writing, v. 8, pp. 1-12.

FERRIS, D. (2002). Treatment of error in second language student writing. Michigan: The University of Michigan Press.

FERRIS, D. (2003). Response to Student Writing: Implications for Second Language Students. Mahwah, NJ: Lawrence Erlbaum Associates.

FERRIS, D. (2004). The "grammar correction" debate in L2 writing: Where are we, and where do we go from here? (and what do we do in the meantime?). Journal of Second Language Writing. v. 13,49-62.

FERRIS, D. (2010). Second language writing research and written corrective feedback in SLA Intersections and Practical Applications. Studies in Second Language Acquisition, v. 32 , pp. $181-201$.

HARMER, J. (2007). The practice of English language teaching. London: Longman.

HENDRICKSON, J. (1978). Error correction in foreign language teaching: Recent research and practice. The Modern Language Journal. v. 62, pp. 387-398.

HENDRICKSON, J. (1980). The treatment of error in written work. The Modern Language Journal. v. 64, pp. 216-221.

HYLAND, K.; HYLAND, F. (2006). Feedback on second language students' writing. Language Teaching, v. 39, pp. 83-101.

KRASHEN, S. (1981). Second language acquisition and second language learning. Oxford: Pergamon Press.

KRASHEN, S. (1982). Principles and practices in second language acquisition. Oxford: Pergamon Press.

KRASHEN, S. (1985). The input bypothesis: Issues and implications. London: Longman.

LALANDE, J.F., 1982. Reducing composition errors: an experiment. Modern Language Journal. v. 66, pp. 140-149.

MILTON, J. (2006). Resource-Rich Web-Based Feedback: helping learners become independent writers. In: K. Hyland y F. Hyland (eds.). Feedback in Second Language Writing: Contexts and Issues. Cap. 7. Cambridge: Cambridge University Press.

PIENEMANN, M. (1998). Language Processing and Second Language Development: Processabilitytheory. Amsterdam/Philadelphia: John Benjamins Publishing Company.

SCHMIDT, R. (1994). Deconstructing consciousness in search of useful definitions for applied linguistics. AILA Review. v 11, pp. 11-26. 
SHEEN, Y. (2007). The effect of focused written corrective feedback and language aptitude on ESL learners' acquisition of articles. TESOL Quarterly, v. 41, pp. 255- 283.

SHEEN, Y. (2011). Corrective Feedback Individual Differences and Second Language Learning. New York: Springster.

TRUSCOTT, J. (1996). The case against grammar correction in L2 writing classes. Language Learning. v. 46, n² 2, pp. 327-369.

TRUSCOTT, J. (1999). The case for "The case against grammar correction in L2 writing classes": A Response to Ferris. Journal of Second Language Writing. v. 8, n², pp. 111 122.

TRUSCOTT, J. (2004). Evidence and conjecture on the effects of correction: a response to Chandler. Journal of Second Language Writing. v. 13, pp. 337-343.

TRUSCOTT, J. (2007). The effect of error correction on learners' ability to write accurately. Journal of Second Language Writing. v. 16, n 4, pp. 255-272.

TRUSCOTT, J. (2009). Arguments and appearances: a response to Chandler. Journal of Second Language Writing. v. 18, pp. 59-60.

VAN BEUNINGEN, C. (2010). Corrective Feedback in L2 Writing: Theoretical Perspectives, Empirical Insights, and Future Directions. International Journal of English Studies. v. $10, n^{\circ} 2$, pp. $1-27$.

VAN BEUNINGEN, C., DE JONG, N.H. y KUIKEN, F. (2008) The effect of direct and indirect corrective feedback on L2 learners' written accuracy. ITL International Journal of Applied Linguistics. v. 156, pp. 279-296.

Recebido: 22/07/2013

Aceito: 17/04/2014 